\title{
Le cancer \\ et ses gènes \\ insaisissables
}

> Pendant près d'un siècle, la théorie des mutations somatiques (somatic mutation theory) a été la théorie dominante utilisée pour expliquer la cancérogenèse. Selon cette théorie, l'accumulation des mutations dans le génome d'une cellule normale unique est responsable des transformations d'une telle cellule en un néoplasme. Il y est implicitement sous-entendu que l'état par défaut des cellules des métazoaires est la quiescence, et que le cancer est une maladie génétique et moléculaire de la cellule. À partir des leçons que nous avons tirées de nos propres recherches sur le contrôle de la prolifération cellulaire, nous avons adopté en 1999 une perspective organiciste, et proposé une théorie concurrente, celle du champ d'organisation tissulaire (tissue organization field theory). $\varepsilon n$ opposition à la théorie des mutations somatiques, la théorie du champ d'organisation tissulaire défend les notions selon lesquelles (1) le cancer est une maladie des tissus, où les cancérigènes (directement) et les mutations dans la lignée germinale (indirectement) peuvent altérer les interactions normales entre le stroma et l'épithélium adjacent, (2) l'état par défaut de toutes les cellules est la prolifération et la motilité, prémisse compatible avec la théorie de l'évolution. Les arguments théoriques et les résultats expérimentaux sont présentés afin de comparer la manière dont les deux théories proposent des principes organisationnels capables d'expliquer objectivement la cancérogenèse. <

\section{Carlos Sonnenschein ${ }^{1}$, Ana M. Soto ${ }^{1,2}$}



«Il est difficile de faire comprendre quelque chose à un homme lorsque son salaire dépend précisément du fait qu'il ne la comprenne pas. »

Upton Sinclair, écrivain

\author{
${ }^{1}$ Department of anatomy and \\ cellular biology, Tufts University \\ School of Medicine, Boston, \\ États-Unis ; \\ ${ }^{2}$ chaire Blaise Pascal 2013-2014, \\ Centre Cavaillès, ENS, Paris, \\ France. \\ carlos.sonnenschein@tufts.edu \\ ana.soto@tufts.edu \\ Texte traduit de l'anglais par \\ A.C. Zielinska \\ a.c.zielinska@gmail.com
}

\section{Les préliminaires historiques et épistémologiques}

Conformément au dogme central de la biologie moléculaire, l'ADN « code » les protéines, et non pas les phénotypes. Néanmoins, l'affirmation grotesque de l'existence d'un lien entre un gène unique et l'homosexualité, l'infidélité ou d'autres traits est encore fréquemment posée dans des journaux scientifiques. De leur côté, les médias renforcent l'idée selon laquelle nous sommes inexorablement déterminés par nos gènes, et par ceux-ci exclusivement. Le déterminisme génétique et le réductionnisme centré sur les gènes ont dominé la pensée biologique depuis 1945, année de la publication du livre d'E. Schrödinger, What is Life, puis la publication en 1971 de l'ouvrage de Jacques Monod, Le Hasard et la nécessité. Cette perspective place le gène dans le «siège du pilote» métaphorique en conceptualisant le développement embryonnaire comme le simple déploiement d'un programme écrit dans nos gènes. Ce dogme ignore des différences incommensurables entre l'équipement génétique d'un organisme et sa complexité phénotypique. Par exemple, le nombre de gènes dans le génome est trop faible pour prédéterminer le développement de l'embryon jusqu'à l'âge adulte. En outre, il n'y a pas de correspondance directe entre le « gène » sur la molécule d'ADN, les brins d'ARN produits à partir de celui-ci et les protéines qui en résultent [3]. En conséquence, ni le réductionnisme ni le déterminisme génétique ne pourront rendre compte des faits empiriques. L'organicisme, qui propose une approche intégrée et dynamique de la biologie, a mis en évidence les faiblesses implicites du déterminisme génétique $[4,5]$.

Les six articles publiés dans ce numéro de médecine/sciences regroupent des ventions faites dans le cadre d'un colloque intitulé «La recherche sur le cancer : un champ privilégié pour penser les rapports entre hasard, rédutionnisme et holisme », organisé le 7 novembre 2013, ENS-Paris, 45, rue d’Ulm, 75005 Paris, par le groupe de travail HAREDHOL (hasard, réductionnisme, holisme). L'« organicisme » inclut des concepts centraux pour l'embryologie du $\mathrm{XIX} \mathrm{X}^{\mathrm{e}}$ siècle et du début du $\mathrm{XX}$, tels que la détermination environnementale 
du phénotype et le polyphénisme. Au cours du $x x^{e}$ siècle, la situation a changé et l'embryologie s'est concentrée sur les animaux qui se reproduisent tout au long de l'année, quand la lumière, la température et la nourriture sont maintenues constantes. Les aspects génétiques ont ainsi été maximisés, alors que la place des facteurs environnementaux a perdu de sa centralité [6].

Les biologistes moléculaires classiques répondent à la résurgence de l'idée d'une détermination environnementale du phénotype en reconnaissant, à contrecœur, que les programmes génétiques sont modulés par l'environnement. C'est l'épigénétique qui est alors présentée comme fournissant une solution. II ne s'agit pas toutefois de l'épigénétique de Waddington, mais d'une version imprégnée d'un essentialisme génétique.

\section{Le réductionnisme peut-il expliquer la complexité biologique?}

La théorie cellulaire stipule que la cellule est l'unité de la vie. Cependant, une cellule au sein d'un organisme multicellulaire ne peut pas prétendre à une existence autonome en dehors de la totalité qu'elle contribue à composer. Les organismes et leurs cellules sont ontogénétiquement liés, en ce sens que les niveaux d'organisation biologique sont entremêlés, et ce, dès le stade de la cellule œuf. En effet, le zygote est à la fois une cellule et un organisme [7]. Dans une perspective réductionniste, les cellules « font » l'organisme grâce à la prolifération cellulaire. Dans la perspective holiste, opposée à celle-ci, l'organisme-zygote fait les cellules par sa division, et, de ce fait, la causalité est descendante. Cependant, l'organicisme voit les embryons comme des systèmes dynamiques ouverts, où l'on retrouve des causalités multiples, ascendantes, descendantes et transversales [7], et où les forces physiques déterminent la forme et la taille des tissus et des organes. Notons que les oppositions entre visions organiciste et réductionniste de la biologie se retrouvent également dans le champ de la cancérogenèse.

\section{Cancer : lexique et définitions}

Deux théories rivales tentent d'expliquer la cancérogenèse : la théorie des mutations somatiques (somatic mutation theory, SMT) reflète le projet déterministe fondé sur les gènes ; la théorie du champ d'organisation tissulaire (tissue organization field theory, TOFT) introduit la notion d'organisme et souligne sa capacité à s'auto-organiser en tant qu'élément conceptuel central de la biologie du cancer [8].

Les cancers gardent la structure distinctive de leurs organes d'origine, tout en étant des «caricatures » des tissus normaux. Les caractéristiques principales du cancer sont, d'une part, une organisation tissulaire altérée, et, d'autre part, une accumulation locale excessive de cellules. Depuis le XIX ${ }^{e}$ siècle, les cancers sont diagnostiqués par les pathologistes en scannant des échantillons de tissu avec des microscopes optiques. Les biologistes moléculaires, qui voient le cancer comme une maladie «moléculaire » et génétique, ont promis de le diagnostiquer un jour à l'aide de l'analyse génomique ; cette promesse attend encore son accomplissement.
Les cancers (ou les néoplasmes) peuvent être aussi bien des tumeurs solides, comme les cancers du sein, de la prostate ou des poumons, que des tumeurs «fluides», comme les cancers des tissus qui composent le sang. Du point de vue clinique, les néoplasmes peuvent être bénins, donc habituellement sans danger pour la survie, ou malins, quand ils deviennent invasifs et métastatiques. Parmi les cancers cliniques, $90 \%$ sont des carcinomes, autrement dit des cancers du tissu épithélial. Les $10 \%$ qui restent sont des néoplasmes du tissu conjonctif, nerveux ou musculaire. Enfin, les premières étapes de la cancérogenèse peuvent soit progresser vers la néoplasie complète et provoquer la mort du patient, soit régresser spontanément ou encore entrer en dormance. Tout cela signifie que la progression et/ou la mort ne sont pas des conséquences inévitables des cancers [8].

\section{Classification des cancers selon leurs causes directes}

Les cancers peuvent être classés en deux groupes distincts : (1) les erreurs innées du développement (inborn errors of development) et (2) les cancers sporadiques (environ $98 \%$ de tous les cancers). Parmi les erreurs innées du développement, on distingue ensuite (a) les erreurs innées du développement héritées, et (b) les erreurs innées du développement acquises. Le premier groupe représente moins de $2 \%$ des cancers cliniques [9] et comprend les rétinoblastomes liés aux mutations du gène $R B$, ainsi que les cancers ovariens et mammaires liés aux gènes $B R C A 1$ et $B C R A 2$. Les erreurs innées du développement acquises apparaissent au moment où des organismes génétiquement normaux sont exposés à des agents environnementaux lors de la gestation, ce qui produit des effets nocifs sur l'organogenèse. Le carcinome à cellules claires du vagin et la néoplasie du sein qui sont le résultat de l'exposition iatrogénique au diéthylstilbestrol, l'œstrogène synthétique, illustrent ce dernier groupe. Notons toutefois que le pourcentage des cancers sporadiques dus à une organogenèse altérée acquise lors de la gestation reste inconnu. Les cancers sporadiques sont provoqués par l'exposition aux cancérigènes pendant la vie post-natale (le tabagisme, la consommation d'alcool, les infections, etc.).

Il existe des cancérigènes physiques (comme les radiations, les corps étrangers), chimiques (comme la fumée du tabac, les contaminants extérieurs) et biologiques (comme Helicobacter pylori, les schistosomes, les papilloma virus humains, etc.). Alors que certains cancérigènes sont mutagènes, d'autres ne le sont pas. L'immense majorité des cancers peuvent être prévenus 
par la réduction de l'exposition aux cancérigènes à tous les âges. Du point de vue sociopolitique, l'échec de la gestion de ces causes environnementales n'est rien de moins que le témoin d'une déréliction des obligations des pouvoirs politiques.

\section{Les causes du cancer et les explications avancées}

La plupart des causes directes des cancers sporadiques sont environnementales. Afin d'expliquer comment ces causes disparates peuvent générer des néoplasmes, la théorie des mutations somatiques suggère que les cancers apparaissent au niveau cellulaire $[10,11]$, alors que la théorie du champ d'organisation tissulaire situe plutôt la cancérogenèse au niveau tissulaire de l'organisation biologique [8]. Selon la théorie des mutations somatiques, le cancer résulte d'une prolifération anormale de cellules générée par l'accumulation des mutations au sein d'une cellule unique $(\rightarrow)$ [32] ; les cancers sont donc clonaux. Pour faire concorder la théorie des mutations somatiques avec la pratique $\rightarrow$ Voir l'article de $\varepsilon$. Solary, page 683 de ce numéro clinique et avec des données expérimentales souvent discordantes, des corrections ad hoc y ont été périodiquement ajoutées. Néanmoins, la théorie des mutations somatiques continue à être enseignée dans les universités du monde entier comme la théorie de référence, et, de ce fait, sa popularité se reflète dans le discours de tous les jours. On entend souvent dire que «le cancer c'est de famille » (cancer runs in the family), ce qui suggère une cause génétique, tout en ignorant que cette famille a pu être exposée à un même environnement nocif (la fumée des cigarettes, les perturbateurs endocriniens, l'amiante, etc.) Inversement, la théorie du champ d'organisation tissulaire postule que la cancérogenèse, tout comme l'histogenèse et l'organogenèse, sont des phénomènes supra-cellulaires se déroulant au niveau tissulaire ${ }^{l}$. Dans cette perspective, le cancer est un «développement qui a mal tourné ». Une autre différence cruciale entre les deux théories concerne les prémisses adoptées dans le contexte du contrôle de la prolifération des cellules et de la motilité des organismes multicellulaires. L'état par défaut est défini en tant qu'état des cellules ayant accès à l'abondance de nutriments. Pour la théorie des mutations somatiques, la quiescence proliférative serait l'état par défaut [12]. La théorie du champ d'organisation tissulaire, quant à elle, en s'appuyant sur des fondements épistémologiques et empiriques, stipule que l'état par défaut de toutes les cellules est la prolifération et la motilité, ce qui est pertinent du point de vue de l'évolution, l'idée étant admise pour les organismes unicellulaires et pour les plantes. Dans ce cas, l'architecture altérée des tissus rend possible l'expression de l'état par défaut, et donc la croissance tumorale et les métastases $[9,13]$.

L'inadéquation croissante entre la théorie des mutations somatiques et les faits empiriques a donc exigé l'ajout de corrections au fur et à mesure du temps à cette théorie. Depuis qu'en 1982, un gène muté unique a été déclaré comme suffisant pour générer une tumeur [14],

${ }^{1}$ Voir le numéro de médecine/sciences «Microenvironnements tumoraux : conflictuels et complémentaires », publié en avril $2014\left(\mathrm{~m} / \mathrm{s} 2014\right.$, vol. $\left.30, \mathrm{n}^{\circ} 4\right)$. le nombre de ces gènes mutés cruciaux a augmenté sans cesse. Pour s'accommoder de cette tournure inattendue des événements, les recherches demandent un effort gargantuesque de séquençage, qui devrait être réalisé sur 2000 tumeurs pour chacun des 50 types tumoraux, correspondant à environ 100000 tumeurs [15]. Par ce séquençage «massivement parallèle », les mutations des cellules cancéreuses ont été arbitrairement classées comme putativement causales (mutations drivers ou conductrices) et comme non pertinentes (mutations passengers) [16]. La distinction entre ces deux types de mutations est ainsi fondée sur des inférences non vérifiables, étant donné qu'il s'agissait là de cancers déjà développés. Il est alors pertinent de signaler ici que des mutations somatiques (silencieuses) ont été identifiées dans les cellules des tissus normaux [17, 18]. Les données les plus frappantes, qui réfutent probablement définitivement la théorie des mutations somatiques, viennent de la publication d'une étude récente qui n'a identifié aucune mutation somatique dans un certain type de tumeurs humaines, les épendymomes [19].

La théorie du champ d'organisation tissulaire déclare explicitement que les «agents cancérigènes génèrent une perturbation dans les interactions entre cellules qui maintiennent l'organisation cellulaire, la réparation des tissus et l'homéostasie locale » [9]. Cette architecture altérée permet aux cellules des tumeurs d'exprimer leur état par défaut, c'est-à-dire la prolifération et la motilité (en formant les métastases). Ce qui se produit au sein des cellules individuelles de la tumeur est donc une conséquence, et non une cause, de cette communication altérée entre les tissus. Selon la théorie du champ d'organisation tissulaire, les mutations représentent un épiphénomène non pertinent du point de vue de la cancérogenèse et de sa progression $[13,20]$.

\section{L'intersection du cancer et de la biologie}

T. Dobzhansky a remarqué que «rien en biologie n'a de sens, si ce n'est à la lumière de l'évolution», et le cancer n'est pas une exception à cette règle. En suivant la tradition organiciste, nous avons interrogé expérimentalement la question de la nature des cibles de la cancérogenèse: sont-elles les cellules épithéliales ou le stroma (tissu conjonctif) de la glande mammaire ? عn utilisant une stratégie neutre quant au cadre théorique, nous avons observé que des combinaisons constituées de stromas exposés à un cancérogène, auxquels on ajoute des cellules épithéliales normales et non exposées, devenaient des néoplasmes [21]. La combinaison inverse (où des cellules épithéliales normales sont exposées à un cancérogène, alors que le stroma ne 
l'est pas) ne conduisait pas à la formation de néoplasme. En conséquence, c'est plutôt le stroma et non pas les cellules individuelles de l'épithélium, qui semble être la cible des cancérigènes. Ensuite, nous avons testé la possibilité de «normaliser » le phénotype cancéreux en combinant des cellules de carcinome mammaire avec du stroma normal: nous avons observé que les cellules tumorales généraient alors des canaux mammaires phénotypiquement normaux [22]. Ces données sont en accord avec les attentes de la théorie du champ d'organisation tissulaire et remettent en question la théorie des mutations somatiques. La normalisation des phénotypes néoplasiques est à présent confirmée par des outils qui identifient de façon univoque les cellules normales qui ont pourtant été cancéreuses autrefois [23].

\section{La science, la société et l'énigme du cancer}

Quatre décennies après la déclaration de la guerre contre le cancer, qui concentrait tous ses efforts sur la théorie des mutations somatiques, même ses défenseurs les plus fervents reconnaissent que cette guerre est perdue et que le retour sur investissement n'est pas au rendez-vous [24]. Il est grand temps de repenser les investissements futurs dans la recherche sur le cancer, en se fiant cette fois-ci à la stratégie fondée sur des prémisses pertinentes du point de vue de l'évolution.

La perspective que nous proposons modifie les priorités de recherche dans le domaine du cancer, et plus largement en biologie [25]. Premièrement, la renaissance de la biologie des systèmes, discipline dont les racines se trouvent dans l'école viennoise de von Bertalanffy et de Weiss [26], où l'organicisme et les causalités à la fois ascendantes et descendantes sont tous pris en compte, ouvre la possibilité de comprendre des phénomènes complexes [27, 28]. Deuxièmement, les nouvelles disciplines d'ingénierie de tissus et la recherche sur les biomatériaux, originalement prévues pour produire des tissus à des fins de transplantation, ont fait renaître l'intérêt de la biophysique pour les études de morphogenèse dans le développement et dans le cancer [29]. Troisièmement, les philosophes et les biologistes théoriques signalent plusieurs difficultés posées par des métaphores trompeuses qui, d'une part, attribuent aux gènes le contenu d'information, et, d'autre part, au génome la capacité de «programmer» l'organisme [30]. Ainsi, l'exploration de visions alternatives anticipe un changement plus qu'attendu dans la recherche en biologie au sens large, et celle sur le cancer en particulier $[31,33](\rightarrow)$. Nous soutenons que les métaphores d'information devraient être remplacées par une théorie solidement fondée sur la physique, capable de produire des données

$\rightarrow$ Voir l'article de J.P. Capp, page 693 de ce numéro

observationnelles nouvelles pour la quantification et pour la compréhension de l'organisation biologique [30]. Cela équivaudrait en somme à proposer une théorie des organismes. $\diamond$

\section{SUMMARY}

Cancer and the elusive cancer genes

For almost a century, the somatic mutation theory (SMT) has been the prevalent theory to explain carcinogenesis. The SMT posits that the accumulation of mutations in the genome of a single normal cell is responsible for the transformation of such cell into a neoplasm. Implicitly, this theory claims that the default state of cells in metazoan is quiescence and that cancer is a cell-based, genetic and molecular disease. From lessons learned while performing our own research on control of cell proliferation and while adopting an organicist perspective, in 1999, we proposed a competing theory, the tissue organization field theory (TOFT). In contraposition to the SMT, (1) the TOFT posits that cancer is a tissue-based disease whereby carcinogens (directly) and mutations in the germ-line (indirectly) may alter normal interactions between the stroma and their adjacent epithelium. And (2) the TOFT explicitly acknowledges that the default state of all cells is proliferation and motility, a premise that is relevant to and compatible with evolutionary theory. Theoretical arguments and experimental evidence are presented to compare the merits of the original SMT and its variants and those of the TOFT in organizing principles, construct objectivity, and ultimately explain carcinogenesis. $\diamond$

\section{REMERCIEMENTS}

Ce travail a été soutenu par des financements de la Bradshaw Foundation, de la Parsemus Foundation, de la Great Neck Breast Cancer Coalition, de la Babylon Breast Cancer Coalition, de l'Italian Space Agency (ASI), de l'Avon Foundation et des National Institutes of Environmental Health Sciences (ES08314 and ES013884).

\section{LIENS D'INTÉRÊT}

Les auteurs déclarent n'avoir aucun lien d'intérêt concernant les données publiées dans cet article.

\section{RÉFÉRENCES}

1. Schrödinger $\varepsilon$. What is life? The physical aspect of the living cell. Cambridge : The University Press, 1945. Qu'est-ce que la vie? De la physique à la biologie, traduit de l'anglais par Léon Keffler. Paris : Seuil, 1993.

2. Monod J. Le hasard et la nécessité : essai sur la philosophie naturelle de la biologie moderne, Paris : Éditions du Seuil, 1970. Chance and necessity: an essay on the natural philosophy of modern biology. New York : Knopf, 1971.

3. Hull D. The Philosophy of biological science. Englewood Clifts NJ : Prentice Hall, 1974.

4. Gilbert SF, Sarkar S. Embracing complexity: organicism for the $21^{\text {st }}$ century. Dev Dyn $2000 ; 219$ : 1-9.

5. Soto AM, Sonnenschein C. Emergentism as a default: cancer as a problem of tissue organization. J Biosci $2005 ; 30: 103-18$.

6. Gilbert SF. Mechanisms for the environmental regulation of gene expression: ecological aspects of animal development. J Biosci $2005 ; 30: 65-74$.

7. Soto AM, Sonnenschein C, Miquel PA. On physicalism and downward causation in developmental and cancer biology. Acta Biotheor 2008 ; 56 : 257-74.

8. Sonnenschein C, Soto AM. The society of cells: cancer and control of cell proliferation. New York : Springer Verlag, 1999.

9. Sonnenschein C, Soto AM. Theories of carcinogenesis: an emerging perspective. Semin Cancer Biol 2008 ; 18 : 372-7. 


\section{RÉFÉRENCES}

10. Boveri T. The origin of malignant tumors. Baltimore, MD : Williams and Wilkins, 1929.

11. Soto AM, Sonnenschein C. One hundred years of somatic mutation theory of carcinogenesis: is it time to switch? BioEssays $2014 ; 36: 118-20$

12. Alberts B, Johnson A, Lewis J, et al. Molecular biology of the cell, $5^{\text {th }}$ ed. London : Garland Science, 2008.

13. Soto AM, Sonnenschein C. The tissue organization field theory of cancer: a testable replacement for the somatic mutation theory. BioEssays $2011 ; 33: 332-40$.

14. Shih C, Shilo BZ, Goldfarb MP, et al. Passage of phenotypes of chemically transformed cells via transfection of DNA and chromatin. Proc Natl Acad Sci USA $1979 ; 76: 5714-8$.

15. Lawrence MS, Stojanov P, Mermel CH, et al. Discovery and saturation analysis of cancer genes across 21 tumour types. Nature 2014 ; $505: 495-501$.

16. Greenman C, Stephens $P$, Smith, R, et al. Patterns of somatic mutation in human cancer genomes. Nature 2007 ; $446: 153-8$.

17. Duncan AW, Hanlon Newell AE, Smith L, et al. Frequent aneuploidy among human hepatocytes. Gastroenterology $2011 ; 142: 25-8$.

18. Yurov YB, lourov IY, Vorsanova SG, et al. Aneuploidy and confined chromosomal mosaicism in the developing human brain. PLoS One 2007 ; 2 : e558.

19. Mack SC, Witt H, Piro RM, et al. Epigenomic alterations define lethal CIMP-positive ependymomas of infancy. Nature $2014 ; 506: 445-50$.

20. Sonnenschein C, Soto AM. The death of the cancer cell. Cancer Res $2011 ; 71: 4334-7$.

21. Maffini MV, Soto AM, Calabro JM, et al. The stroma as a crucial target in rat mammary gland carcinogenesis. J Cell Sci 2004 ; 117 : 1495-502.

22. Maffini MV, Calabro JM, Soto AM, Sonnenschein C. Stromal regulation of neoplastic development: age-dependent normalization of neoplastic mammary cells by mammary stroma. Am J Pathol $2005 ; 167: 1405-10$.

23. Booth BW, Boulanger CA, Anderson LH, Smith GH. The normal mammary microenvironment suppresses the tumorigenic phenotype of mouse mammary tumor virus-neu-transformed mammary tumor cells. Oncogene $2011 ; 30: 679-89$.

24. Hanahan D. Rethinking the war on cancer. Lancet 2014 ; 383 : 585-63.

25. Sonnenschein C, Soto AM. Why systems biology and cancer? Semin Cancer Biol 2011 ; 21 : 147-9.

26. Drack $M$, Wolkenhauer 0 . System approaches of Weiss and Bertalanffy and their relevance for systems biology today. Semin Cancer Biol $2011 ; 21: 150-5$.

27. Saetzler K, Sonnenschein C, Soto AM. Systems biology beyond networks: generating order from disorder through self-organization. Semin Cancer Biol 2011 ; 21 : 165-74.

28. Sasai Y. Cytosystems dynamics in self-organization of tissue architecture. Nature $2013 ; 493$ : 318-26.

29. Ingber $D E$, Levin $M$. What lies at the interface of regenerative medicine and developmental biology? Development $2009 ; 134: 2541-7$.

30. Longo G, Miquel PA, Sonnenschein C, Soto AM. Is information a proper observable for biological organization? Prog Biophys Mol Biol 2012 ; 109 : 108-14.

31. Bizzarri M, Giuliani A, Cucina A, et al. Fractal analysis in a systems biology approach to cancer. Semin Cancer Biol $2011 ; 21: 175-82$.

32. Solary $\varepsilon$. Une approche réductionniste du cancer. Med Sci (Paris) $2014 ; 30: 683-7$.

33. Capp JP. Le rôle des phénomènes aléatoires dans le cancer. Med Sci (Paris) 2014 ; $30: 693-8$

\section{Autres articles de ce numéro relatifs au colloque}

- Zielinska A, Issad T. La recherche sur le cancer : un champ privilégié pour penser les rapports entre hasard, réductionnisme et holisme. Med Sci (Paris) $2014 ; 30: 675-8$.

- Morange M. Les modèles explicatifs du cancer : aspects historiques. Med Sci (Paris) $2014 ; 30: 679-82$.

- Solary $\varepsilon$. Une approche réductionniste du cancer. Med Sci (Paris) 2014 ; $30: 683-7$.

- Capp JP. Le rôle des phénomènes aléatoires dans le cancer. Med Sci (Paris) $2014 ; 30: 693-8$.

- Kupiec JJ. Comment le hasard intervient-il dans le débat entre holisme et réductionnisme? Conclusion du dossier Cancer/Haredhol. Med Sci (Paris) $2014 ; 30: 699-700$.

\section{POUR EN SAVOIR PLUS (sélection d'articles de $\mathrm{m} / \mathrm{s}$ )}

- Microenvironnements tumoraux : conflictuels et complémentaires. Med Sci (Paris) $2014 ; 30: 343-466$.

- Fridman WH, Sautès-Fridman C. Le microenvironnement tumoral. Med Sci (Paris) $2014 ; 30: 359-65$.

- Provot S. Contrôle de la croissance et de la dissémination tumorales par le microenvironnement. Med Sci (Paris) 2014 ; 30 : 366-71.

- Le Guellec S, Duprez-Paumier R, Lacroix-Triki M. Microenvironnement tumoral : la vision du pathologiste. Med Sci (Paris) 2014 ; 30 : 372-7.

- Buache $\varepsilon$, Rio MC. Le stroma tumoral : un terreau fertile pour la cellule cancéreuse. Med Sci (Paris) $2014 ; 30: 385-90$.

-Stoppa-Lyonnet D, Houdayer C. Séquençage de nouvelle génération en génétique médicale. Med Sci (Paris) 2012 ; 28 : 123-4.

- Jordan B. Chroniques génomiques. Des signatures sans valeur? Med Sci (Paris) $2011 ; 28: 547-50$.

- Jordan B. Chroniques génomiques. L'insoutenable complexité des cancers. Med Sci (Paris) 2011 ; 27 : 781-6.

- Jordan B. Chroniques génomiques. Séquençage de nouvelle génération: déjà en clinique ? Med Sci (Paris) 2011 ; 27 : 1127-30.

- Bertucci F, Finetti P, Cervera N, Birnbaum D. Classification pronostique du cancer du sein et profils d'expression génique sur puces à AND. Med Sci (Paris) $2008 ; 24: 599-606$.

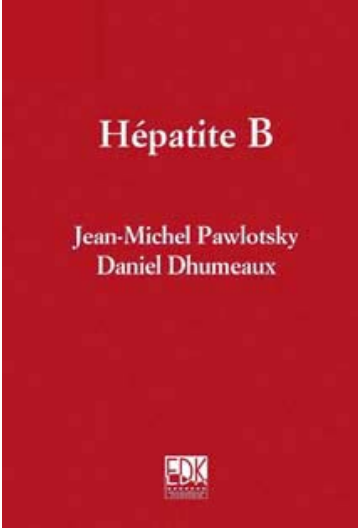

ISBN : 978-2-8425-4131-6 576 pages

\section{Bon de commande}

À retourner à EDK, 2, rue Troyon - 92316 Sèvres Cedex

Tél. : 0155641393 - Fax : 0155641394 - E-mail : edk@edk.fr

NOM :

Prénom :

Adresse :

Code postal :

Ville :

Pays :

Fonction :

Je souhaite recevoir l'ouvrage Hépatite B : $54 €+3 €$ de port $=\mathbf{5 7} €$ TTC offre exceptionnelle réservée aux abonnés à $\mathrm{m} / \mathrm{s}$ jusqu'au 31 décembre 2010

en .............. exemplaire, soit un total de ............................... €
$\square$ Par chèque, à l'ordre de $\mathbf{E} \mathbf{D}$ K
$\square$ Par carte bancaire : $\quad \square$ Visa $\square$ Eurocard/Mastercard

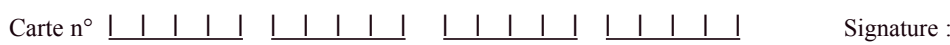

Date d'expiration: $\quad \leq 1|\underline{1}|$

$\mathrm{N}^{\circ}$ de contrôle au dos de la carte : $\quad$ |
TIRÉS À PART

C. Sonnenschein 